\title{
Morphism Properties of Digital Categories
}

\author{
Simge Öztunç* \\ Celal Bayar University Faculty of Arts and Science, Departments of Mathematics, \\ Şehit Prof. Dr. İlhan Varank Campus, Manisa/TURKEY \\ simge.oztunc@cbu.edu.tr \\ *Corresponding Author/
}

Received: 8 December 2016

Accepted: 15 May 2017

DOI: $10.18466 /$ cbayarfbe.339314

\begin{abstract}
In this paper we defined the $\mathrm{Img}_{\kappa}$ category and researched the properties of monomorphism, epimorphism and isomorphism for digital categories which are related with the categorical structure in [1]. Also initial and terminal objects in digital categories are defined by using $\kappa-$ adjacency relation. Hence we determined the initial and terminal objects of digital categories which have digital image with $\kappa-$ adjacency as objects. In addition to this we proved that the objects of the same type in a digital category are isomorphic.

Keywords - Digital Image, Digital Category, $\kappa-$ monomorphism, $\kappa-$ epimorphism, $\kappa-$ isomorphism.
\end{abstract}

\section{Introduction}

Digital Topology is a field of mathematical science which investigates the image processing and digital image processing. Many Researchers, for example Rosenfeld [2], Han [3], Kong [4], Boxer [5,6], Karaca [7] and others have contributed this area with their research. The notion of digital image, digital continuous map is studied in $[2,3,4,5,6]$. Their characterization and effective computation became a useful tool for our research. Then we carry this notion to category theory and we construct some fundamental category models in digital topology.

We introduce the $\operatorname{Img}_{\kappa}$ Category and give the monomorphism and epimorphism properties of $\operatorname{Img}_{\kappa}$ category. Also we define the initial and terminal objects in $\operatorname{Img}_{\kappa}$ and prove that initial and terminal objects are isomorphic in $\operatorname{Img}_{\kappa}$.

\section{Preliminaries}

In this study we indicate the set of integers by $\square$. Then $\square^{n}$ denotes the set of lattice points in Euclidean $n$-dimensional spaces. A finite subset of $\square^{n}$ with an adjacency relation is said to be digital image.

\section{Definition 2.1. ([5] and [6 ])}

(1) Two points $p$ and $q$ in $\square$ are 2 -adjacent if $|p-q|=1$.

(2) Two points $p$ and $q$ in $\square^{2}$ are 8 -adjacent if they are distinct and differ by at most 1 in each coordinate.

(3) Two points $p$ and $q$ in $\square^{2}$ are 4 -adjacent if they are 8 -adjacent and differ by exactly one coordinate.

(4) Two points $p$ and $\boldsymbol{q}$ in $\square^{3}$ are 26-adjacent if they are distinct and differ by at most 1 in each coordinate.

(5) Two points $p$ and $q$ in $\square^{3}$ are 18-adjacent if they are 26-adjacent and differ in at most two coordinates.

(6) Two points $p$ and $q$ in $\square^{3}$ are 6-adjacent if they are 18 -adjacent and differ by exactly one coordinate.

Suppose that $\kappa$ be an adjacency relation defined on $\square^{n}$ . A digital image $X \subset \square^{n}$ is $\kappa$-connected [5] if and only if for every pair of points $\{x, y\} \subset X, x \neq y$, there 
is a set $\left\{x_{0}, x_{1}, \ldots, x_{c}\right\} \subset X$ such that $x=x_{0}, x_{c}=y$ and $x_{i}$ and $x_{i+1}$ are $\kappa$-neighbors, $i \in\{0,1, \ldots, c-1\}$.

Definition 2.2. Let $X$ and $Y$ are digital images such that $X \subset \square^{n_{0}}, \quad Y \subset \square^{n_{1}}$. Then the digital function $f: X \rightarrow Y$ is a function which is defined between digital images.

Definition 2.3. ([5] and [6]) Let $X$ and $Y$ are digital images such that $X \subset \square^{n_{0}}, \quad Y \subset \square^{n_{1}}$. Assume that $f: X \rightarrow Y$ be a function. Let $\kappa_{i}$ be an adjacency relation defined on $\square^{n_{i}}, i \in\{0,1\} . f$ is called to be $\left(\kappa_{0}, \kappa_{1}\right)$-continuous if the image under $f$ of every $\kappa_{0}$-connected subset of $X$ is $\kappa_{1}$-connected .

A function satisfying Definition 2.3 is referred to be digitally continuous. A consequence of this definition is given below.

Proposition 2.4. ([5] and [6]) Let $X$ and $Y$ are digital images. Then the function $f: X \rightarrow Y$ is said to be $\left(\kappa_{0}, \kappa_{1}\right)$-continuous if and only if for every $\left\{x_{0}, y_{0}\right\} \subset X$ such that $x_{0}$ and $x_{1}$ are $\kappa_{0}$-adjacent, either $f\left(x_{0}\right)=f\left(x_{1}\right)$ or $f\left(x_{0}\right)$ and $f\left(x_{1}\right)$ are $\kappa_{1}$-adjacent .

The basic notions of Category Theory is given in [8], [9]. Also digital and soft properties of categories are investigated in [1], [10].

Definition 2.5. [1] A category $C$ consists of the following data:

- Objects: $A, B, C, \ldots$

- Arrows: $f, g, h, \ldots$

- For each arrow $f$, there are given objects $\operatorname{dom}(f), \operatorname{cod}(f)$

called the domain and codomain of $f$. We write

$$
f: A \rightarrow B
$$

to indicate that $A=\operatorname{dom}(f)$ and $B=\operatorname{cod}(f)$

- Given arrows $f: A \rightarrow B$ and $g: B \rightarrow C$, that is, with

there is given an arrow

$$
\operatorname{cod}(f)=\operatorname{dom}(g)
$$

$$
g \circ f: A \rightarrow C
$$

called the composite of $f$ and $g$.
- $\quad$ For each object $A$, there is given an arrow

$$
1_{A}: A \rightarrow A
$$

called the identity arrow of $A$.

This data required to satisfy the following laws:

Associativity: $\quad h \circ(g \circ f)=(h \circ g) \circ f \quad$ for $\quad$ all $f: A \rightarrow B, g: B \rightarrow C$ and $h: C \rightarrow D$.

Unit: $f \circ 1_{A}=f=1_{B} \circ f$ for all $f: A \rightarrow B$.

\section{The Category $\operatorname{Img}_{\kappa}$}

- The objects of $\operatorname{Img}_{\kappa}$ are $\kappa$-adjacent digital images,

- The morphisms of $\operatorname{Img}_{\kappa}$ are $\kappa$-continuous (digitally continuous) functions,

- $\quad$ For each morphism $f$ in $\operatorname{Img}_{\kappa}$

$$
\operatorname{dom}(f) \text { and } \operatorname{cod}(f)
$$

are $\kappa$-adjacent digital images.

- Let $(A, \kappa),(B, \kappa)$ and $(C, \kappa)$ be $\kappa$-adjacent digital images, i.e., objects in $\mathrm{Img}_{\kappa}$. Given morphisms $\quad f:(A, \kappa) \rightarrow(B, \kappa) \quad$ and $g:(B, \kappa) \rightarrow(C, \kappa)$ with $\operatorname{cod}(f)=\operatorname{dom}(g)$. Then the function $g \circ f:(A, \kappa) \rightarrow(C, \kappa)$ is a $\kappa$-continuous function, since the composition of two digital continuous ( $\kappa$-continuous ) is continuous. Thus $g \circ f:(A, \kappa) \rightarrow(C, \kappa)$ an $\operatorname{Img}_{\kappa}-$ morphism.

- For each $(A, \kappa), \operatorname{Img}_{\kappa}$ - object, the identity map $1_{(A, \kappa)}:(A, \kappa) \rightarrow(A, \kappa)$ is a $\kappa$-continuous function. Therefore $1_{(A, \kappa)}$ is an $\operatorname{Img}_{\kappa}$ - morphism.

- Composition:

For all $\quad f:(A, \kappa) \rightarrow(B, \kappa)$, $g:(B, \kappa) \rightarrow(C, \kappa)$ and $h:(C, \kappa) \rightarrow(D, \kappa)$ $\mathrm{Img}_{\kappa}-$ morphisms, we have $h \circ(g \circ f)=(h \circ g) \circ f$

- Identity:

For each $f:(A, \kappa) \rightarrow(B, \kappa) \quad \operatorname{Img}_{\kappa}-$ morphism

$$
f \circ 1_{(A, \kappa)}=f=1_{(B, \kappa)}
$$

Thus we have construct the $I m g_{\kappa}$ category of digital images and digital continuous functions.

Let now investigate some properties of $\mathrm{Img}_{\kappa}$ category. 


\section{Epis and Monos in $\operatorname{Img}_{\kappa}$}

Definition 4.1: Let $(A, \kappa),(B, \kappa)$ and $(C, \kappa)$ be Img $_{\kappa}$ - objects which have the same cardinality. For given any $h, g:(C, \kappa) \rightarrow(A, \kappa) \operatorname{Img}_{\kappa}-$ morphism, if $f \circ g=f \circ h$ implies that $g=h$, then

$$
f:(A, \kappa) \rightarrow(B, \kappa)
$$

$\mathrm{Img}_{\kappa}-$ morphism is called a $\kappa-$ monomorphism.

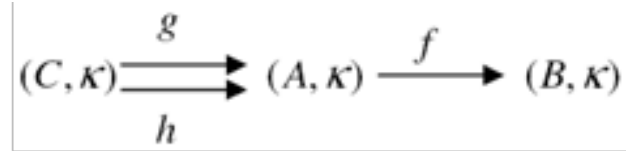

Definition 4.2: Let $(A, \kappa),(B, \kappa)$ and $(D, \kappa)$ be $\operatorname{Img}_{\kappa}$ - objects which have the same cardinality. For given any $i, j:(B, \kappa) \rightarrow(D, \kappa) \operatorname{Img}_{\kappa}-$ morphism, if $i \circ f=j \circ f$ implies that $i=j$, then

$$
f:(A, \kappa) \rightarrow(B, \kappa)
$$

$I m g_{\kappa}-$ morphism is called a $\kappa-$ epimorphism.

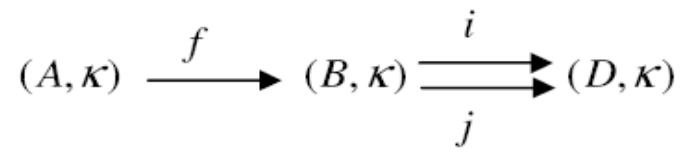

Definition 4.3: In category $I m g_{\kappa}$, an $I m g_{\kappa}-$ morphism

$$
f:(A, \kappa) \rightarrow(B, \kappa)
$$

is said to be an $\kappa$-isomorphism if there is a $g:(B, \kappa) \rightarrow(A, \kappa) \operatorname{Img}_{\kappa}-$ morphism such that

$$
g \circ f=1_{(A, \kappa)} \text { and } f \circ g=1_{(B, \kappa)} .
$$

It is written as $(A, \kappa) \cong(B, \kappa)$.

Lemma 4.4: If $f:(A, \kappa) \rightarrow(B, \kappa) \quad$ and $g:(B, \kappa) \rightarrow(C, \kappa)$ be two $\kappa-$ monomorphisms in $\operatorname{Img}_{\kappa}$ category, then $g \circ f:(A, \kappa) \rightarrow(C, \kappa)$ is a $\kappa-$ monomorphism.

Proof: Let $\quad f:(A, \kappa) \rightarrow(B, \kappa) \quad$ and
$g:(B, \kappa) \rightarrow(C, \kappa)$ be two $\kappa-$ monomorphisms. Suppose that $h, k:(C, \kappa) \rightarrow(A, \kappa)$ are $\operatorname{Img}_{\kappa}-$ morphisms. If

then it follows that

$$
(g \circ f) \circ h=(g \circ f) \circ k
$$

$$
g \circ(f \circ h)=g \circ(f \circ k)
$$

from the associativity property of $I m g_{\kappa}$-morphisms. Since $g$ is an $\kappa$-monomorphism, we obtain that $f \circ h=f \circ k$. On the other hand, since $f$ is an $\kappa-$ monomorphism, we have $h=k$. Therefore $g \circ f$ is a $\kappa-$ monomorphism.

Lemma 4.5: If $f:(A, \kappa) \rightarrow(B, \kappa) \quad$ and $g:(B, \kappa) \rightarrow(C, \kappa)$ be two $\kappa-$ epimorphisms in $\operatorname{Img}_{\kappa}$ category, then $g \circ f:(A, \kappa) \rightarrow(C, \kappa)$ is a $\kappa-$ epimorphism.

Proof: Let $\quad f:(A, \kappa) \rightarrow(B, \kappa) \quad$ and $g:(B, \kappa) \rightarrow(C, \kappa)$ be two $\kappa-$ epimorphisms. Assume that $h, k:(B, \kappa) \rightarrow(D, \kappa)$ are $\operatorname{Img}_{\kappa}-$ morphisms. If

$$
h \circ(g \circ f)=k \circ(g \circ f) \text {, }
$$

We have

$$
(h \circ g) \circ f=(k \circ g) \circ f
$$

From the associativity property. Since $f$ is an $\kappa$ - epimorphism, we obtain that $h \circ g=k \circ g$, and since $g$ is an $\kappa-$ epimorphism, we get $h=k$. Consequently $g \circ f$ is a $K$ - epimorphism.

Theorem 4.6: Every isomorphism in $\operatorname{Img}_{\kappa}$ is both $\kappa-$ monomorphism and $\kappa-$ epimorphism.

Proof: Consider the following diagram:

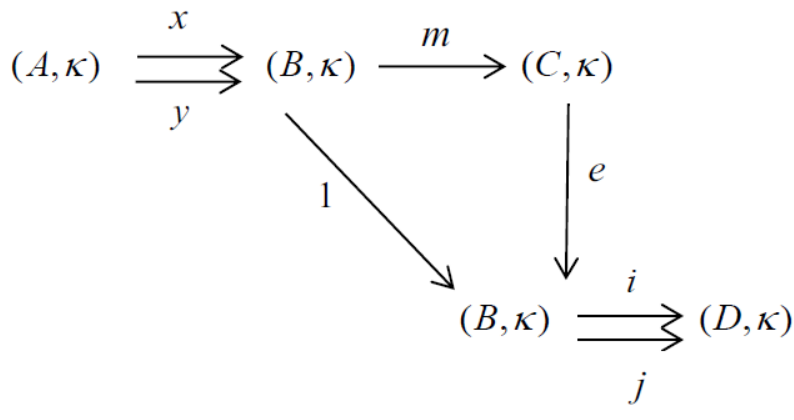




$$
i \circ e \circ m=j \circ e \circ m=i \circ 1_{(B, \kappa)}=j \circ 1_{(B, \kappa)} .
$$

Thus we obtain that $i=j$. Consequently $m$ is a $\kappa-$ epimorphism.

Definition 4.7: An object $(U, \kappa)$ of a category $\operatorname{Img}_{\kappa}$ is said to be an initial object if for every object $(X, \kappa)$ of $I m g_{\kappa}$, the set $\operatorname{Mor}_{I m g_{\kappa}}((U, \kappa),(X, \kappa))$ is a singeleton. Dually $(U, \kappa)$ is said to be a terminal object if for every object $(X, \kappa)$ of $\operatorname{Img}_{\kappa}$, the set $\operatorname{Mor}_{\text {Img }_{\kappa}}((X, \kappa),(U, \kappa))$ is a singeleton.

Theorem 4.8: Let $\mathrm{Img}_{\kappa}$ be a category of digital images and digital continuous functions. Then any two initial objects in $I m g_{\kappa}$ are isomorphic.

Proof: Let $\left(U_{1}, \kappa\right)$ and $\left(U_{2}, \kappa\right)$ be initial objects in $\operatorname{Img}_{\kappa}$. We must show $\operatorname{Mor}_{\operatorname{Img}_{\kappa}}\left(\left(U_{1}, \kappa\right),\left(U_{2}, \kappa\right)\right)$ contains an isomorphism. If $\left(U_{1}, \kappa\right)$ is an initial object for $\operatorname{Img}_{\kappa}$, then $\operatorname{Mor}_{\operatorname{Img}_{\kappa}}\left(\left(U_{1}, \kappa\right),\left(U_{2}, \kappa\right)\right)=\{\alpha\}$ for any object $\left(U_{2}, \kappa\right)$. If $\left(U_{2}, \kappa\right)$ is an initial object for $I m g_{\kappa}$, then $\operatorname{Mor}_{\text {Img }_{\kappa}}\left(\left(U_{2}, \kappa\right),\left(U_{1}, \kappa\right)\right)=\{\beta\}$ for any object $\left(U_{1}, \kappa\right)$. We get $\alpha \circ \beta \in \operatorname{Mor}_{\text {Img }_{\kappa}}\left(\left(U_{2}, \kappa\right),\left(U_{2}, \kappa\right)\right)$ by the composition property of morphisms. Therefore $\alpha \circ \beta=i d_{U_{2}}$. Similarly we have $\beta \circ \alpha=i d_{U_{1}}$. It follows that $\alpha$ is an isomorphism with $\beta=\alpha^{-1}$ and $\left(U_{1}, \kappa\right)$ and $\left(U_{2}, \kappa\right)$ are isomorphic.

Theorem 4.9: Let $\mathrm{Img}_{\kappa}$ be a category of digital images and digital continuous functions. Then any two terminal objects in $\operatorname{Img}_{\kappa}$ are isomorphic.

Proof: Assume that $\left(O_{1}, \kappa\right)$ and $\left(O_{2}, \kappa\right)$ are terminal objects in $\operatorname{Img}_{\kappa}$. We want to show that $\operatorname{Mor}_{\text {Img }_{\kappa}}\left(\left(O_{1}, \kappa\right),\left(O_{2}, \kappa\right)\right)$ contains an isomorphism. If $\left(O_{1}, \kappa\right)$ is a terminal object for $\operatorname{Img}_{\kappa}$, then $\operatorname{Mor}_{\text {Img }_{\kappa}}\left(\left(O_{2}, \kappa\right),\left(O_{1}, \kappa\right)\right)=\{f\}$ for any object $\left(\mathrm{O}_{2}, \kappa\right)$. If $\left(\mathrm{O}_{2}, \kappa\right)$ is a terminal object for $\operatorname{Img}_{\kappa}$, then $\operatorname{Mor}_{\text {Img }_{\kappa}}\left(\left(O_{1}, \kappa\right),\left(O_{2}, \kappa\right)\right)=\{g\}$ for any object $\left(O_{1}, \kappa\right)$. We have $g \circ f=i d_{\left(O_{2}, \kappa\right)}$ and similarly $f \circ g=i d_{\left(O_{1}, \kappa\right)}$ by the composition property of morphisms. Therefore $f$ is an isomorphism with $f=g^{-1}$ and $\left(O_{1}, \kappa\right)$ and $\left(O_{2}, \kappa\right)$ are isomorphic.

Corollary 4.10: The objects of the same type in $\mathrm{Img}_{\kappa}$ are isomorphic.

Proof: The proof is obtained by Theorem 4.8 and Theorem 4.9.

\section{Conclusion}

We have construct the category $\operatorname{Img}_{\kappa}$ and worked on some properties of $\mathrm{Img}_{\kappa}$ in this paper. We have obtained some foldings deal with monomorphism and epimorphism properties of $\mathrm{Img}_{\kappa}$. Also we concluded that the objects of the same type in the category $\mathrm{Img}_{\kappa}$ are isomorphic.

\section{References}

1. Öztunç S., Mutlu A., Categories in Digital Images, American Journal of Mathematics and Statistics, January 2013;Vol.3, No.1.

2. Rosenfeld A., 'Continuous' functions on digital pictures, Pattern Recognition Letters, 1986; Vol. 4, 177-184.

3. Han, S.E. An Extended Digital $01\left(\mathrm{k}_{0}, \mathrm{k}_{1}\right)$-Continuity. $J$. Appl. Math. Comput. 2004, 16, 445-452.

4. Kong T.Y., Roscoe A.W., and Rosenfeld A., Concepts of digital topology, Topology and its Applications, 1992, 46, 219-262.

5. Boxer L., Digitally continuous functions, Pattern Recognition Letters, 1994, 15 833-839.

6. Boxer L., Properties of Digital Homotopy, Journal of Mathematical Imaging and Vision, 2005, 22 19-26.

7. Karaca I., Boxer L. and Öztel A., Topological Invariants in Digital Images, Jour. of Mathematical Sciences: Advances and Applications, 2011, 2, 109-140.

8. Awoday S., Category Theory, Oxford Science Publication, 2010.

9. Blyth T.S., Categories, Longman 1986.

10. Öztunç S., Some Properties of Soft Categories, International Journal of Modeling and Optimization, 2016; 6(2),91-95. 